\title{
One-Carbon Metabolism and Colorectal Cancer: Potential Mechanisms of Chemoprevention
}

\author{
Matthew P. Hanley • Daniel W. Rosenberg
}

Published online: 21 March 2015

(C) Springer International Publishing AG 2015

\begin{abstract}
For many years, folic acid has been evaluated for its utility as a chemopreventive agent due to its position at the center of the one-carbon metabolic network. This network is responsible for generating precursors to nucleotide synthesis as well as the one-carbon moieties used in DNA methylation reactions, two mechanisms which are frequently disrupted during carcinogenesis. While the use of folic acid for the chemoprevention of colorectal cancer is still controversial, there is evidence that folic acid intake has significant influence on these fundamental cellular mechanisms. Folic acid has a dual role with regards to nucleotide synthesis and colorectal cancer prevention; in a healthy colon, adequate folate status is important for nucleotide metabolism homeostasis and the maintenance of DNA integrity; however, in a colon harboring premalignant lesions, lowered folate status may help to eliminate transformed cells. In addition, folic acid is important for the generation of the one-carbon groups used in DNA methylation reactions, and modulation of folic acid metabolism may be useful in combating the aberrant DNA methylation during carcinogenesis. Interestingly, it has been revealed that decreased folic acid intake can dampen the inflammatory response, which has recently been a popular strategy for colorectal cancer chemoprevention. In this review, we discuss the molecular mechanisms influenced by folic acid intake and how they might be relevant to cancer chemoprevention in greater detail.
\end{abstract}

This article is part of the Topical Collection on Cancer Chemoprevention

M. P. Hanley • D. W. Rosenberg $(\bowtie)$

Center for Molecular Medicine, University of Connecticut Health

Center, 263 Farmington Avenue, Farmington, CT 06032-3103, USA

e-mail: rosenberg@uchc.edu
Keywords Folic acid · Folate $\cdot$ Colorectal cancer . Chemoprevention · Methyl donor · Nucleotide synthesis . DNA methylation $\cdot$ Inflammation

\section{Introduction}

Over the last few decades, there has been increased interest in how an array of dietary components may affect cancer progression and whether any of these components have potential for use as chemopreventative agents. Folate, also known as vitamin $\mathrm{B}_{9}$, has been the subject of numerous studies seeking to understand its role in cancer progression [1-6]. This is due, in part, to the 1996 mandate by the United States Food and Drug Administration (FDA) requiring the supplementation of wheat, rice, bread, cereals, pasta, and other grain products with folic acid in an effort to reduce the incidence of neural tube defects in newborns [7]. Following this mandate, several studies were published that put forth conflicting findings regarding the influence of folate supplementation on colorectal cancer (CRC) progression; nearly two decades later, the debate over whether folate promotes or prevents CRC continues. The controversy surrounding the influence of folate on tumorigenesis is likely due to its position at the center of a complex metabolic network that influences a variety of biological processes including nucleotide synthesis, DNA integrity, and gene expression through epigenetic regulation [2]. The most thoroughly characterized function of folate is its immediate role in one-carbon metabolism. Through a series of metabolic reactions, dietary folate can be converted into several forms, each of which plays a role in distinct metabolic pathways. The three most thoroughly investigated folate metabolites are 5methyl tetrahydrofolate (THF), 10-formyl THF, and 5,10methylene THF. These three metabolites all provide onecarbon groups for use in a variety of biosynthesis reactions (Fig. 1) [8]. 10-Formyl THF and 5,10-methylene THF are key 
a

Folate One-Carbon Metabolism

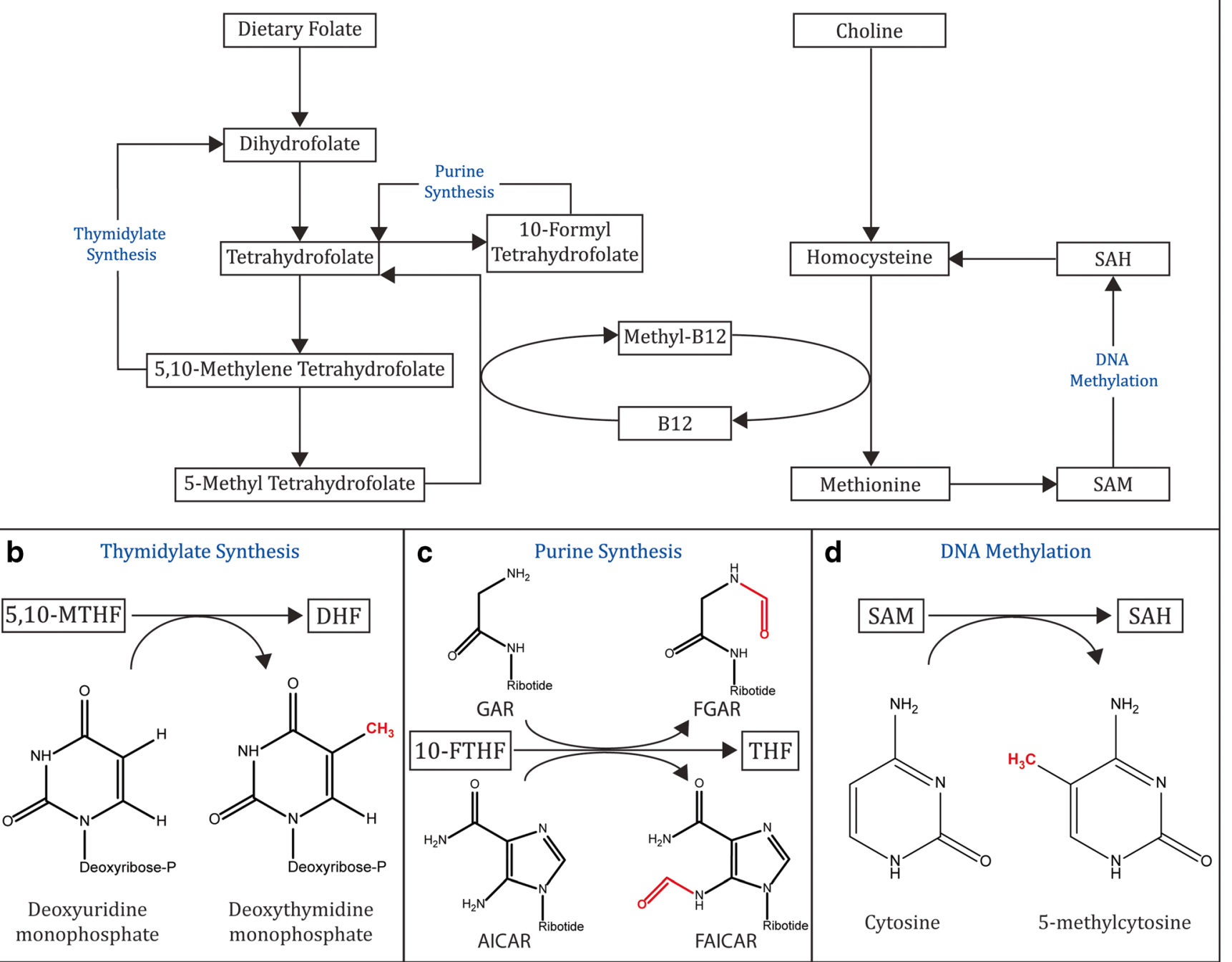

Fig. 1 a Overview of folate metabolism. Dietary folate is converted into several key metabolites by a series of enzymatic conversions. b 5,10Methylene tetahydrofolate is used as the substrate for thymidylate synthesis, where a methyl group is donated to deoxyuridine monophosphate (dUMP) to form deoxythymidine monophosphate (dTMP). c 10-Formyl tetrahydrofolate performs a similar function in the synthesis of the purine nucleotide precursor, inosine monophosphate (IMP). Initially, 10-FTHF donates a formyl group to convert glycinamide ribonucleotide (GAR) to $N$-formylglycinamide ribonucleotide (FGAR).

intermediates required for thymidylate and pyrimidine biosynthesis, respectively, while 5-methyl THF feeds one-carbon groups into the homocysteine metabolic cycle, the pathway which serves as the primary source of methyl groups used in DNA methylation reactions [9]. It is because of folate's involvement in many biological processes relevant to cancer development, and its potential to modulate these processes, that it has been widely considered for its potential chemopreventive benefit. However, despite significant potential and a large body of research into its multifaceted functions, understanding of the intricate
In a later step, 10-FTHF donates a formyl group to convert 5Aminoimidazole-4-carboxamide ribonucleotide (AICAR) into 5formamidoimidazole-4-carboxamide ribotide (FAICAR). d Methyl groups can be shuttled from 5-methyl tetrahydofolate (5-MTHF) to the homocysteine cycle, where they are used to regenerate the "universal methyl donor," $S$-adenosyl methionine (SAM). SAM in turn serves as the substrate for the DNA methyltransferase enzymes, which transfer a methyl group from SAM to cytosine, resulting in the formation of 5methylcytosine

effects of folate intake and supplementation on cancer risk remains elusive.

\section{Folic Acid and Colorectal Cancer Risk}

A large number of studies, both preclinical and clinical, have sought to understand how intake of folic acid influences tumor progression; a collection of key studies can be found in Table 1. Unfortunately, this body of work has not yet been able to provide a clear picture of how folate intake influences 


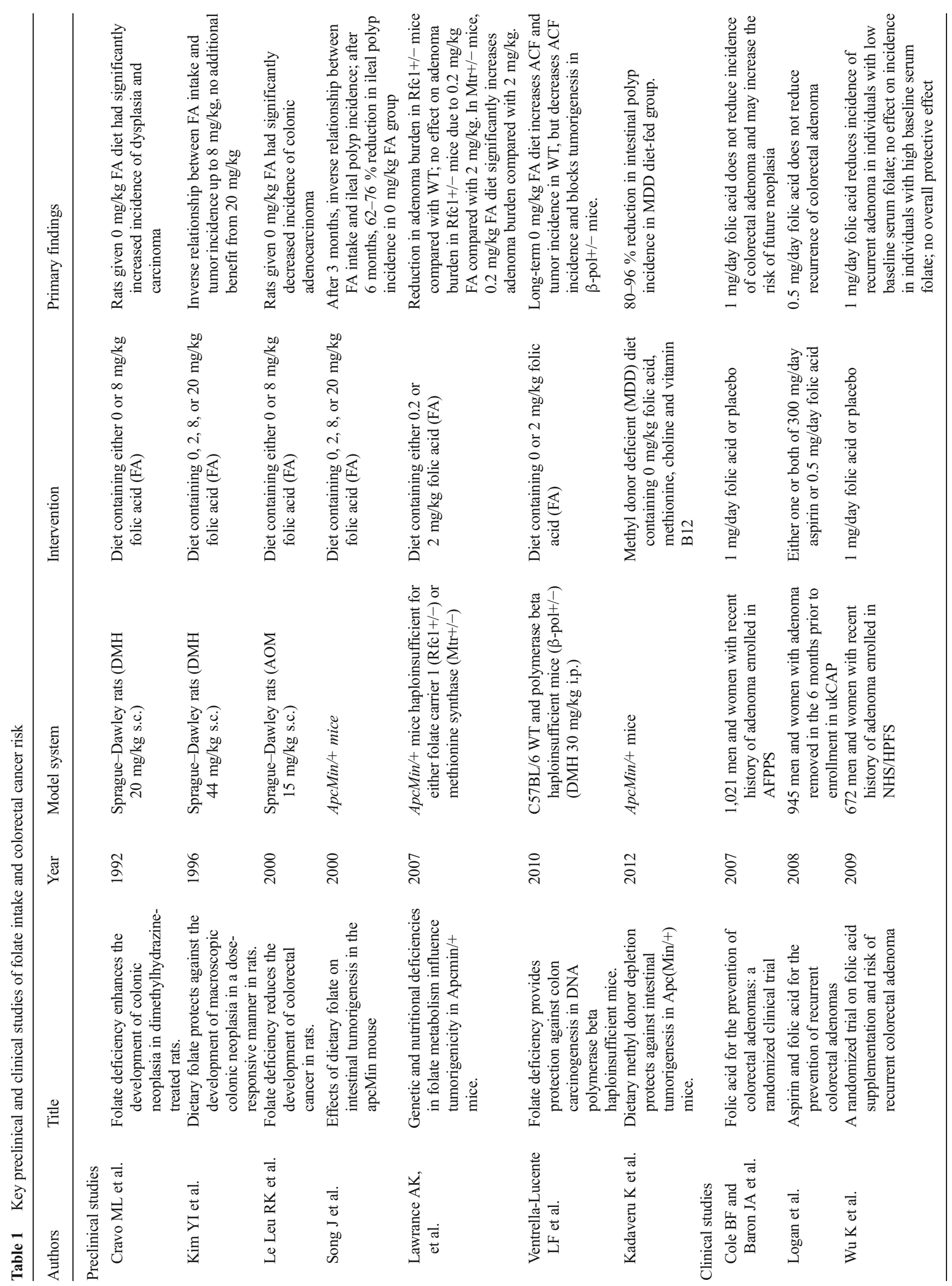


colorectal cancer risk. The earliest investigations into the relationship between folate and CRC risk were typically epidemiological case-control studies examining associations between CRC incidence and folate intake determined from dietary questionnaires. Three such studies that were among the first to report on folate intake and CRC risk demonstrated an inverse relationship between folate consumption and colorectal cancer incidence [2, 10-12]. Importantly, all three of these studies were conducted in countries that, at the time, had not implemented folic acid fortification programs and were therefore more likely to have larger portions of the population with inadequate folate intake. For the most part, studies of folic acid intake and the risk of colorectal neoplasia conducted in the United States after the implementation of the national fortification program have also supported an inverse relationship between intake and risk in the general population [13-15]. However, a key randomized clinical trial conducted by Cole and Baron [16] challenged this long-standing paradigm by demonstrating that very high doses of folic acid $(1 \mathrm{mg} /$ day $)$ in individuals with a history of colorectal adenoma do not decrease the risk of future adenoma and may actually result in an increased risk. Additionally, a more recent meta-analysis of six large folic acid supplementation trials has concluded that folic acid supplementation is associated with an increased risk of cancer $[17 \bullet \bullet$.

Studies in rodent models have suggested that once neoplastic lesions have been established folate deficiency slows tumor progression $\left[18,19,2^{\bullet}\right]$. As a result, it has been suggested that folic acid has a "dual modulatory effect" on carcinogenesis [21]; prior to the establishment of neoplastic lesions, high folate intake decreases the risk of cancer, while high folate intake after the establishment of neoplasia drives progression. Thus, within the context of folate-based chemoprevention strategies, careful consideration of dosage and timing with respect to carcinogenic initiation are critical determinants of the safety and efficacy of an intervention.

\section{Folic Acid as a Carbon Source in De Novo Nucleotide Synthesis}

Folic acid metabolites are used as substrates in the biosynthesis of both thymidylate and purine nucleotides. Once folate has entered the cell, it is reduced to tetrahydrofolate (THF), which is the derivative that acts as the central nexus of the folic acid metabolic cycle. THF goes through a series of enzymatic conversions, with each step in the pathway giving rise to an intermediate that serves a unique role. The most important folate derivatives for nucleotide metabolism are 5,10-methylene THF and $\mathrm{N}^{10}$-formyl THF. 5,10-methylene THF is the substrate for the enzyme thymidylate synthase which transfers a methyl group from 5,10-methylene THF to deoxyuridine monophosphate (dUMP) to generate thymidine monophosphate (dTMP) [22]. $\mathrm{N}^{10}$-Formyl THF, on the other hand, is the substrate for two enzymes involved in separate steps in the biosynthesis of purine nucleotides: Phosphoribosylglycinamide formyltransferase (GART) and phosphoribosylaminoimidazolecarboxamide formyl transferase (AICARFT) [23, 24]. GART transfers a methyl group from $\mathrm{N}^{10}$-formyl THF to glycinamide ribonucleotide (GAR) to generate the purine intermediate $\mathrm{N}$-formylglycinamide ribonucleotide. Later in the purine biosynthetic pathway, AICARFT transfers another carbon from $\mathrm{N}^{10}$-formyl THF to AICAR to generate the purine intermediate 5-formamidoimidazole-4-carboxamide ribonucleotide (Fig. 1) [25]. Thus, the supply of folate is incredibly important for nucleotide biosynthesis, as it is the primary source of crucial structural carbons in purine precursors and of the carbon that is necessary to convert dUMP to dTMP. Imbalances in these key folate metabolites have previously been detected in colorectal tumors, underscoring the relevance of this metabolic pathway to colon carcinogenesis [26].

It is folic acid's role in nucleotide metabolism that has made it an interesting chemopreventive candidate from a mechanistic standpoint. It has been hypothesized that folate deficiency causes DNA damage by reducing the availability of nucleotide synthesis precursors and thus causing an imbalance in nucleotide pools [27]. Such imbalances are known to lead to uracil misincorporation into DNA, DNA mismatches, and double-stranded breaks [27, 28]. Inhibition of one-carbon metabolism, such as that caused by dietary folate deficiency, has been shown to cause uracil misincorporation and DNA double-stranded breaks in lymphocytes and colonic epithelial cells in rodent models [29-31]. It has also previously been shown that dietary folate deficiency is capable of inducing both uracil misincorporation and chromosome breaks in human DNA [32,33]. Thus, when folate status is low in a normal cell, that cell will accumulate DNA damage and have a higher probability of undergoing transformation; on the one hand, folic acid supplementation has been proposed to reduce CRC risk by ensuring the ample supply of nucleotide precursors. On the other hand, this same mechanism might help to explain the inhibitory effect of folate deficiency on tumor growth. Cells which have undergone transformation tend to have a higher rate of replication and a higher demand for nucleotides than their comparatively slow-replicating normal counterparts. In these transformed cells, low folate status places a severe restriction on nucleotide biosynthesis, inhibiting DNA replication and slowing tumor growth [34]. A recent study by Witherspoon [35 ${ }^{\circ}$ revealed that the chemopreventive agent $\alpha$-difluoromethylornithine (DFMO) exerts its protective effects through a similar mechanism; DFMO alters the metabolic availability of folate derivatives and inhibits thymine biosynthesis.

It is possible that transformed cells are unable to cope with the rapid accumulation of DNA damage caused by folate 
deficiency and eventually activate apoptotic pathways, resulting in the death of the initiated cell before it can develop into a more advanced lesion. In fact, this mechanism has been used for many years as the basis for antimetabolite chemotherapy; 5-fluorouracil (5-FU) acts by inhibiting thymidylate synthase which leads to the reduced availability of thymidylate, inhibition of DNA replication, and ultimately, the induction of apoptosis [36]. Furthermore, antifolate drugs such as methotrexate and pemetrexed function by inhibiting steps in folate one-carbon metabolism and have consequences for DNA integrity that are similar to those of 5-FU [37].

Confusion regarding the effects of folate intake on CRC risk may arise from the fact that these two effects of deficiency are not necessarily mutually exclusive. An individual with low folate intake may have two distinct subpopulations of colonic epithelial cells: normal cells which are sustainin DNA damage and are pushed towards transformation and a population of initiated cells the progression of which is inhibited by folate restriction. For the purpose of chemoprevention, then, a folate-based intervention may take one of two forms depending on an individual's personal risk profile. Endoscopic detection of an adenoma is a known risk factor for future neoplasia, suggesting that individuals with a history of adenoma may possess multiple synchronous neoplastic foci capable of progressing to a more advanced lesion. In these cases, it may be of greater benefit to the patient to restrict folate intake so as to inhibit the progression of existing neoplasia; in a young individual with a colon free of neoplastic foci, it may be of greater benefit to supplement folate intake in order to prevent DNA damage and reduce the likelihood of neoplastic initiation. A key task which must be completed in order to maximize the utility of folate chemoprevention is the characterization of the effects of various levels of folate intake on cellular viability at different stages of carcinogenesis. From these data, it may be possible to identify individuals who belong to specific at-risk subpopulations that stand to benefit most from folate supplementation or restriction. The overall effect on carcinogenesis of a given folate intake level may be quite different for certain high-risk individuals, such as patients with a personal history of colorectal cancer or individuals with familial adenomatous polyposis, than it is for the general population.

\section{Folic Acid as a Source of One-Carbon Groups for DNA Methylation}

A second mechanism through which folate intake has been proposed to offer chemopreventive effects is the modulation of DNA methylation patterns. In a landmark paper, Feinberg and Vogelstein established the connection between genomic hypomethylation and colorectal carcinogenesis by demonstrating that, compared with matched normal tissue, primary tumor samples were significantly hypomethylated at a specific subset of genes [38]. This finding was expanded upon when Feinberg later demonstrated that, compared with normal mucosa, adenomas and adenocarcinomas have reductions in total genomic 5-methylcytosine content of $8 \%$ and $10 \%$, respectively [39]. A link between hypomethylation and carcinogenesis was later demonstrated in an animal model when Gaudet found that mice expressing hypomorphic Dnmt1 developed aggressive lymphomas between 4 and 8 months of age [40]. It was later revealed that, while tumors do undergo a global reduction in DNA methylation, they also become hypermethylated at specific $\mathrm{CpG}$ islands in a tissue-specific manner, suggesting that there exists a more general relationship between the disruption of normal methylation patterns and colorectal carcinogenesis.

The supply of one-carbon groups for use in methylation reactions is dependent on metabolic flux through the homocysteine cycle [41], which generates $S$ adenosyl methionine (SAM), the "universal methyl donor." Dietary folic acid is eventually converted to the metabolite 5methyltetrahydrofolate (5-MTHF); a methyl group is then transferred to homocysteine to generate methionine, which reacts with ATP to generate SAM. SAM is the substrate for DNA methyltransferase enzymes (DNMTs) which transfer a methyl group to DNA CpGs, converting SAM to $S$-adenosyl homocysteine (SAH) and the target cytosine to 5methylcytosine (Fig. 1) [42]. The availability of SAM and SAH are important regulators of DNMT activity and the maintenance of patterns of DNA methylation. Considering the importance of folate for homocysteine metabolism and DNA methylation, it has been suggested that folate-based chemoprevention may help to maintain normal patterns of methylation $[43,44]$.

Studies of the relationship between folic acid intake and DNA methylation status have produced inconsistent results. While some studies have reported that folate supplementation has no effect on global DNA methylation $[33,45,46]$, there is also ample evidence to support a relationship between folate intake and methylation. Several studies have shown that increased folic acid intake is associated with an increase in global DNA methylation in both leukocytes and colonic epithelial cells $[47,48]$. It has also been shown that folate deficiency causes both a global reduction in DNA methylation and specific hypomethylation at the p53 gene promoter in human colon carcinoma cells [43]; it was further demonstrated that both types of hypomethylation could be reversed when folic acid was added to the culture medium. This finding was partially recapitulated in an animal model when $\mathrm{Apc}^{\mathrm{Min} /+}$ mice were fed a diet deficient in folate: folate deficiency was associated with both a decrease in DNA hypomethylation and SAM [49]. Interestingly, in a 2007 study by Keyes [48], this positive correlation between folate intake and global DNA methylation was found to be present in 18-month-old mice 
but not in 4-month-old mice. A more recent study showed that, in old mice (18 months), administration of a diet deficient in folate and supplemented with $18 \%$ ethanol caused a significant reduction in global DNA methylation compared with control [50]. These studies suggest an age-dependent susceptibility of DNA methylation patterns to modulation by folate intake.

There have been only a few clinical studies investigating the relationship between folate intake and DNA methylation in humans, but, in general, they support a positive correlation between folic acid intake and DNA methylation. Early studies indicated that supplementation of folic acid intake caused an increase in DNA methylation in cells of the colonic mucosa $[47,51,52]$. However, one drawback of these early studies is that they all relied on the methyl acceptance assay, which has been reported to be subject to a large degree of variation [53]. Two more recent studies, which measured DNA methylation with more sophisticated techniques, have also produced inconsistent findings; one study confirmed the positive correlation between folate intake and DNA methylation in the colon while the other did not [33, 54].

Importantly, the findings of two key studies may warrant a reevaluation of our understanding of the effects of folate intake on methylation during colorectal carcinogenesis. These studies showed that, when the activity of either Dnmt1 or Dnmt3a is reduced in $A p c^{\mathrm{Min} /+}$ mice, there is a dramatic reduction in intestinal tumor multiplicity; in the case of Dnmt1, tumorigenesis is suppressed completely. These studies suggest that, in the intestine and colon, carcinogenesis is dependent on DNMT activity. It is well known that DNA methylation is critical for regulating chromatin conformation [55], and it is thought that hypomethylation of a particular region of DNA may cause it to be more susceptible to accumulating damage. In the past, it has been proposed that folic acid supplementation might provide chemopreventive protection by preventing hypomethylation and reducing susceptibility to genomic damage [56]. If, however, colorectal carcinogenesis is dependent on methylation, it may actually be beneficial to restrict the generation of methyl groups destined for use in DNA methylation in certain situations. This view of folate-dependent methylation in the context of colorectal cancer may help to explain the anti-tumor effects of folate deficiency that have been recently described in mouse models [19-21]. Just as the effects of folate intake on tumorigenesis appear to vary depending on timing and dosage, they may also vary depending on an individual's age and epigenetic background.

Taken together, the variability in these studies suggests that the effects of folate intake on DNA methylation may be modulated by confounding factors, including age and intake of alcohol. If modulation of methylation patterns is a key mechanism through which folic acid chemoprevention may exert its effects, it will be important to tease out which members of the DNA methylation pathway (SAM, SAH, DNMTs, etc.) are the dominant regulators. In addition, it is possible that, based on their unique epigenetic profile, certain individuals may benefit from folate-based chemoprevention more than others. It will be important to understand how outside factors, such as other dietary factors or advanced age, interact with folatedependent methylation.

\section{Folic Acid as a Requirement for Immune Cell Proliferation}

Although it is not discussed as frequently as biosynthesis or DNA methylation within the context of folate chemoprevention, one-carbon metabolism is important for the maintenance of the immune system and the inflammatory response. There is considerable evidence that inflammation is a significant risk factor for the development of colorectal cancer [57-59]. Much like cancer cells, immune cells divide rapidly following activation and are therefore highly dependent on an adequate supply of nucleotides [60-62]. Thus, it is possible that lowered folate intake and the subsequent restriction of nucleotide availability will inhibit immune cell proliferation and lead to an attenuated inflammatory response. While inhibition of the immune system comes with a host of potential complications, it may serve as the basis for a useful chemopreventive strategy for particular subpopulations, such as individuals with inflammatory bowel disease (IBD). The practice of disrupting one-carbon metabolism as an anti-inflammatory therapy has been in use for some time; the antifolate methotrexate (MTX) has been administered at low doses to treat rheumatoid arthritis for several decades [63]. Investigations into the anti-inflammatory mechanism of MTX showed that it acts by inhibiting replication of the immune cells responsible for mediating the inflammatory response [64]. Mild to moderate deficiency in folic acid intake, in certain subpopulations, may function through the same mechanism to reduce inflammation and cancer risk.

With the exception of a recent study which demonstrated that mouse monocytes cultured in a folate-depleted medium significantly upregulate expression of inflammatory cytokines, most evidence points towards an immunosuppressive effect of folate deficiency. Depletion of folic acid has been shown to inhibit the proliferation of $\mathrm{T}$ cells in vitro [65]; this finding is particularly interesting because ulcerative colitis , a form of IBD which carries a significant increase in CRC risk, is characterized by $\mathrm{T}$ cell infiltration of the colonic mucosa [66]. Studies in animal models and in humans also generally support an anti-inflammatory effect of folic acid deficiency. In order to better understand the role of folate metabolism in the process of tumor development, our laboratory [20•] recently restricted the flux of one-carbon metabolism by administering a diet deficient in the methyl donors folate, choline, methionine, and $\mathrm{B} 12$ to $\mathrm{Apc}^{\mathrm{Min} / \mathrm{+}}$ mice. In this study, it was 
demonstrated that methyl donor deficiency dramatically reduces tumor burden in the small intestine; it was further shown that this reduction in tumor burden was associated with a decrease in immune cell populations and in the expression of inflammatory cytokines. A prior clinical trial in which adult men were placed on a folate-deficient diet for either 4 or 8 weeks showed a similar reduction in inflammatory markers [33]. A study of folic acid intake in the AOM/DSS mouse model of colitis-associated CRC demonstrated that tumor multiplicity was positively correlated with folic acid intake; folate-deficient mice had the lowest number of tumors [67]. Together, these studies demonstrate the potential for a folic acid-based anti-inflammatory intervention, which has important implications for colorectal cancer chemoprevention.

There is considerable evidence to support the use of nonsteroidal anti-inflammatory drugs (NSAIDs) for the chemoprevention of colorectal cancer [68]. Interestingly, it has been shown that a large number of NSAIDs commonly considered for use as chemopreventive agents act as competitive inhibitors of folate-dependent enzymes in addition to their primary mechanisms of action [69]. This observation may suggest that these NSAIDs have chemopreventive efficacy due, in part, to the inhibition of folate one-carbon metabolism and presents the possibility of using antifolate drugs for chemoprevention. It may be worthwhile to reevaluate existing antifolates for use as chemopreventive agents, particularly in high-risk populations such as individuals who have recently undergone colorectal tumor resection and are therefore at a high risk of relapse [70]. Drugs such as methotrexate, which is already used at low dosages for long-term management of rheumatoid arthritis, may be the best candidates to be repurposed for use in chemoprevention.

\section{Conclusion}

Folic acid may still prove to be a useful chemopreventive agent, but it will likely find the most utility when interventions are tailored for an individual based on his personal medical history and unique genetic background. Due to the multiple mechanisms through which the modulation of folic acid intake may exert its chemoprotective effects, and the differences among at-risk populations, it will be necessary to carefully tailor interventions to an individual's unique risk profile. If, for example, folate supplementation promotes the progression of established neoplasia [16], it may be prudent for an individual to reduce folate consumption following endoscopic detection of an adenoma. It is especially important to understand the optimal folate intake for specific high-risk subpopulations considering the FDA-mandated national fortification program and the effects it may have on non-target populations. Though this program achieved its goal of reducing the incidence of neural tube defects by raising folate intake in women of childbearing age, it has concurrently raised folate intake nationwide, with men and women over the age of 50 years being the most significantly affected [71, 72]. Collectively, the data suggest that dietary supplementation with folic acid is not protective against CRC in all instances and that successful folate-based chemoprevention will require better understanding of the optimal intake level for particular groups of people as well as carefully targeted interventions.

While nucleotide biosynthesis is the best-characterized folate-dependent molecular mechanism [73], steps still need to be taken to understand how its role in cancer risk changes throughout the carcinogenic process. As a cell acquires more genomic changes and progresses towards cancer, its metabolic demands change. This should be taken into account when considering a biosynthesis-based mechanism of folate chemoprevention. Similarly, DNA methylation profiles are known to be influenced by a number of outside factors including age and the consumption of alcohol $[50,74,75]$. Thus, not only may different subpopulations require unique chemopreventive strategies, but the best strategy for a particular individual may change over time in order to adapt to changes in diet, age, and personal history of neoplasia. Finally, effects of modulated folate intake which are not commonly considered for chemopreventive use may find relevance in specific high-risk subpopulations, such as the potential use of the anti-inflammatory effects of folate deficiency in individuals with IBD. While folate chemoprevention has not turned out to be the "magic bullet" it was once hoped to be, it still has potential to be an effective strategy when interventions are founded in a mechanistic understanding and tailored to an individual's unique requirements.

\section{Compliance with Ethics Guidelines}

Conflict of Interest Matthew P. Hanley and Daniel W. Rosenberg declare that they have no conflict of interest.

Human and Animal Rights and Informed Consent This article does not contain any studies with human or animal subjects performed by any of the authors.

\section{References}

Papers of particular interest, published recently, have been highlighted as:

- Of importance

•. Of major importance

1. Freudenheim JL, Graham S, Marshall JR, et al. Folate intake and carcinogenesis of the colon and rectum. Int J Epidemiol. 1991;20: 368-74.

2. Meyer F, White E. Alcohol and nutrients in relation to colon cancer in middle-aged adults. Am J Epidemiol. 1993;138:225-36. 
3. White E, Shannon JS, Patterson RE. Relationship between vitamin and calcium supplement use and colon cancer. Cancer Epidemiol Biomarkers Prev. 1997;6:769-74.

4. Giovannucci E, Rimm EB, Ascherio A, et al. Alcohol, low-methionine-low-folate diets, and risk of colon cancer in men. J Natl Cancer Inst. 1995;87:265-73.

5. Bird CL, Swendseid ME, Witte JS, et al. Red cell and plasma folate, folate consumption, and the risk of colorectal adenomatous polyps. Cancer Epidemiol Biomarkers Prev. 1995;4:709-14.

6. Kim Y-I, Fawaz K, Knox T, et al. Colonic mucosal concentrations of folate correlate well with blood measurements of folate status in persons with colorectal polyps. Am J Clin Nutr. 1998;68:866-72.

7. Health claims and label statements; folate and neural tube defects. Final rule. (Codified in Section 101.79, Title 21, Code of Federal Regulations). Fed Regist. 61:8752-878.

8. Schmitz JC, Stuart RK, Priest DG. Disposition of folic acid and its metabolites: a comparison with leucovorin. Clin Pharmacol Ther. 1994;55:501-8.

9. Tibbetts AS, Appling DR. Compartmentalization of mammalian folate-mediated one-carbon metabolism. Annu Rev Nutr. 2010;30:57-81. doi:10.1146/annurev.nutr.012809.104810.

10. Benito E, Stiggelbout A, Bosch FX, et al. Nutritional factors in colorectal cancer risk: a case-control study in Majorca. Int J Cancer. 1991;49:161-7. doi:10.1002/ijc.2910490202.

11. Glynn SA, Albanes D, Pietinen P, et al. Colorectal cancer and folate status: a nested case-control study among male smokers. Cancer Epidemiol Biomark Prev Publ Am Assoc Cancer Res Cosponsored Am Soc Prev Oncol. 1996;5:487-94.

12. Paspatis GA, Karamanolis DG. Folate supplementation and adenomatous colonic polyps. Dis Colon Rectum. 1994;37:1340-1.

13. Su LJ, Arab L. Nutritional status of folate and colon cancer risk: evidence from NHANES I epidemiologic follow-up study. Ann Epidemiol. 2001;11:65-72.

14. Wei EK, Giovannucci E, Wu K, et al. Comparison of risk factors for colon and rectal cancer. Int J Cancer J Int Cancer. 2004;108:43342. doi:10.1002/ijc. 11540 .

15. Razzak AA, Oxentenko AS, Vierkant RA, et al. Associations between intake of folate and related micronutrients with molecularly defined colorectal cancer risks in the Iowa Women's Health Study. Nutr Cancer. 2012;64:899-910. doi:10.1080/01635581.2012. 714833.

16. Cole BF, Baron JA, Sandler RS, et al. Folic acid for the prevention of colorectal adenomas: a randomized clinical trial. JAMA J Am Med Assoc. 2007;297:2351-9. doi:10.1001/jama.297.21.2351.

17.• Baggott JE, Oster RA, Tamura T. Meta-analysis of cancer risk in folic acid supplementation trials. Cancer Epidemiol. 2012;36:7881. doi:10.1016/j.canep.2011.05.003. This meta-analysis combined data from six large, prospective, randomized folic acid supplementation trials and demonstrated an increased cancer incidence in the supplementation group.

18. Le Leu RK, Young GP, McIntosh GH. Folate deficiency reduces the development of colorectal cancer in rats. Carcinogenesis. 2000;21: 2261-5.

19. Song J, Medline A, Mason JB, et al. Effects of dietary folate on intestinal tumorigenesis in the $A p c^{\mathrm{Min} /+}$ mouse. Cancer Res. 2000;60:5434-40.

20. Kadaveru K, Protiva P, Greenspan EJ, et al. (2012) Dietary methyl donor depletion protects against intestinal tumorigenesis in $\mathrm{Apc}^{\mathrm{Min} /+}$ mice. Cancer Prev Res Phila Pa 5:911-920. doi:10.1158/ 1940-6207.CAPR-11-0544. This study, conducted by our laborato$r y$, demonstrated that dietary folate deficiency, in the context of deficiency of choline, methionine and vitamin B12, is capable of reducing intestinal tumor burden by greater than $90 \%$ in the $\mathrm{ApC}^{\mathrm{Min} /+}$ mouse model.
21. Kim Y-I. Folate: a magic bullet or a double edged sword for colorectal cancer prevention? Gut. 2006;55:1387-9. doi:10.1136/gut. 2006.095463.

22. Carreras CW, Santi DV. The catalytic mechanism and structure of thymidylate synthase. Annu Rev Biochem. 1995;64:721-62. doi: 10.1146/annurev.bi.64.070195.003445.

23. Zhang Y, Morar M, Ealick SE. Structural biology of the purine biosynthetic pathway. Cell Mol Life Sci CMLS. 2008;65:3699 724. doi:10.1007/s00018-008-8295-8.

24. Bulock KG, Beardsley GP, Anderson KS. The kinetic mechanism of the human bifunctional enzyme ATIC (5-amino-4-imidazolecarboxamide ribonucleotide transformylase/inosine 5 '-monophosphate cyclohydrolase) a surprising lack of substrate channeling. J Biol Chem. 2002;277:22168-74.

25. Hartman SC, Buchanan JM. Nucleic acids, purines, pyrimidines (nucleotide synthesis). Annu Rev Biochem. 1959;28:365-410. doi:10.1146/annurev.bi.28.070159.002053.

26. Odin E, Wettergren Y, Carlsson G, Gustavsson B. Determination of reduced folates in tumor and adjacent mucosa of colorectal cancer patients using LC-MS/MS. Biomed Chromatogr BMC. 2013;27: 487-95. doi:10.1002/bmc.2816.

27. Das SK, Kunkel TA, Loeb LA. Effects of altered nucleotide concentrations on the fidelity of DNA replication. Basic Life Sci. 1985;31:117-26.

28. Richards RG, Brown OE, Sedwick WD. Misincorporation of deoxyuridine in human cells: consequences of antifolate exposure. Basic Life Sci. 1985;31:149-62.

29. Duthie SJ, Grant G, Narayanan S. Increased uracil misincorporation in lymphocytes from folate-deficient rats. Br J Cancer. 2000;83: 1532-7. doi:10.1054/bjoc.2000.1481.

30. Kim YI, Shirwadkar S, Choi SW, et al. Effects of dietary folate on DNA strand breaks within mutation-prone exons of the p53 gene in rat colon. Gastroenterology. 2000;119: 151-61.

31. Goulian M, Bleile B, Tseng BY. Methotrexate-induced misincorporation of uracil into DNA. Proc Natl Acad Sci U S A. 1980;77:1956-60.

32. Blount BC, Mack MM, Wehr CM, et al. Folate deficiency causes uracil misincorporation into human DNA and chromosome breakage: implications for cancer and neuronal damage. Proc Natl Acad Sci U S A. 1997;94:3290-5.

33. Protiva P, Mason JB, Liu Z, et al. Altered folate availability modifies the molecular environment of the human colorectum: implications for colorectal carcinogenesis. Cancer Prev Res Phila Pa. 2011;4:530-43. doi:10.1158/1940-6207.CAPR-10-0143.

34. Mathews CK. DNA precursor metabolism and genomic stability. FASEB J Off Publ Fed Am Soc Exp Biol. 2006;20:1300-14. doi: 10.1096/fj.06-5730rev.

35. Witherspoon M, Chen Q, Kopelovich L, et al. Unbiased metabolite profiling indicates that a diminished thymidine pool is the underlying mechanism of colon cancer chemoprevention by alphadifluoromethylornithine. Cancer Discov. 2013;3:1072-81. doi:10.1158/2159-8290.CD-12-0305. This study demonstrates that $\alpha$-difluoromethylornithine, an agent with demonstrated chemopreventive utility in the colon, functions by inhibiting thymidine biosynthesis. This result lends credence to the hypothesis that dietary restriction of folate, which also inhibits thymidine biosynthesis, may yet have promise as a chemopreventive intervention.

36. Longley DB, Harkin DP, Johnston PG. 5-Fluorouracil: mechanisms of action and clinical strategies. Nat Rev Cancer. 2003;3:330-8. doi:10.1038/nrc1074.

37. Fleisher M. Antifolate analogs: mechanism of action, analytical methodology, and clinical efficacy. Ther Drug Monit. 1993;15: 521-6. 
38. Feinberg AP, Vogelstein B. Hypomethylation distinguishes genes of some human cancers from their normal counterparts. Nature. 1983;301:89-92.

39. Feinberg AP, Gehrke CW, Kuo KC, Ehrlich M. Reduced genomic 5 -methylcytosine content in human colonic neoplasia. Cancer Res. 1988;48:1159-61.

40. Gaudet F, Hodgson JG, Eden A, et al. Induction of tumors in mice by genomic hypomethylation. Science. 2003;300:489-92. doi:10.1126/science.1083558.

41. Stead LM, Au KP, Jacobs RL, et al. Methylation demand and homocysteine metabolism: effects of dietary provision of creatine and guanidinoacetate. Am J Physiol Endocrinol Metab. 2001;281: E1095-100.

42. Hoffman DR, Marion DW, Cornatzer WE, Duerre JA. SAdenosylmethionine and $S$-adenosylhomocystein metabolism in isolated rat liver. Effects of L-methionine, L-homocystein, and adenosine. J Biol Chem. 1980;255:10822-7.

43. Wasson GR, McGlynn AP, McNulty H, et al. Global DNA and p53 region-specific hypomethylation in human colonic cells is induced by folate depletion and reversed by folate supplementation. J Nutr. 2006; 136:2748-53.

44. Jhaveri MS, Wagner C, Trepel JB. Impact of extracellular folate levels on global gene expression. Mol Pharmacol. 2001;60:128895. doi:10.1124/mol.60.6.1288.

45. Duthie SJ, Narayanan S, Brand GM, Grant G. DNA stability and genomic methylation status in colonocytes isolated from methyldonor-deficient rats. Eur J Nutr. 2000;39:106-11.

46. Jung AY, Smulders Y, Verhoef P, et al. No effect of folic acid supplementation on global DNA methylation in men and women with moderately elevated homocysteine. PLoS One. 2011;6:e24976. doi: 10.1371/journal.pone.0024976.

47. Pufulete M, Al-Ghnaniem R, Khushal A, et al. Effect of folic acid supplementation on genomic DNA methylation in patients with colorectal adenoma. Gut. 2005;54:648-53. doi:10.1136/gut.2004. 054718 .

48. Keyes MK, Jang H, Mason JB, et al. Older age and dietary folate are determinants of genomic and p16-specific DNA methylation in mouse colon. J Nutr. 2007;137:1713-7.

49. Sibani S, Melnyk S, Pogribny IP, et al. Studies of methionine cycle intermediates (SAM, SAH), DNA methylation and the impact of folate deficiency on tumor numbers in Min mice. Carcinogenesis. 2002;23:61-5.

50. Sauer J, Jang H, Zimmerly EM, et al. Ageing, chronic alcohol consumption and folate are determinants of genomic DNA methylation, p16 promoter methylation and the expression of $\mathrm{p} 16$ in the mouse colon. Br J Nutr. 2010;104:24-30. doi:10.1017/ S0007114510000322.

51. Cravo ML, Pinto AG, Chaves P, et al. Effect of folate supplementation on DNA methylation of rectal mucosa in patients with colonic adenomas: correlation with nutrient intake. Clin Nutr Edinb Scotl. 1998;17:45-9.

52. Cravo M, Fidalgo P, Pereira AD, et al. DNA methylation as an intermediate biomarker in colorectal cancer: modulation by folic acid supplementation. Eur J Cancer Prev Off J Eur Cancer Prev Organ ECP. 1994;3:473-9.

53. Dahl C, Guldberg P. DNA methylation analysis techniques. Biogerontology. 2003;4:233-50. doi:10.1023/A:1025103319328.

54. Wallace K, Grau MV, Levine AJ, et al. Association between folate levels and $\mathrm{CpG}$ island hypermethylation in normal colorectal mucosa. Cancer Prev Res Phila Pa. 2010;3:1552-64. doi:10.1158/ 1940-6207.CAPR-10-0047.

55. Keshet I, Lieman-Hurwitz J, Cedar H. DNA methylation affects the formation of active chromatin. Cell. 1986;44:535-43. doi:10.1016/ 0092-8674(86)90263-1.
56. Kim Y-I. Folate and carcinogenesis: evidence, mechanisms, and implications. J Nutr Biochem. 1999;10:66-88. doi:10.1016/ S0955-2863(98)00074-6.

57. Greten FR, Eckmann L, Greten TF, et al. IKKbeta links inflammation and tumorigenesis in a mouse model of colitis-associated cancer. Cell. 2004;118:285-96. doi:10.1016/j.cell.2004.07.013.

58. Lakatos P-L, Lakatos L. Risk for colorectal cancer in ulcerative colitis: changes, causes and management strategies. World J Gastroenterol WJG. 2008;14:3937-47.

59. Chan AT, Ogino S, Giovannucci EL, Fuchs CS. Inflammatory markers are associated with risk of colorectal cancer and chemopreventive response to anti-inflammatory drugs. Gastroenterology. 2011;140:799-808. doi:10.1053/j.gastro.2010.11.041. quiz ell.

60. Pizzini RP, Kumar S, Kulkarni AD, et al. Dietary nucleotides reverse malnutrition and starvation-induced immunosuppression. Arch Surg Chic Ill 1960. 1990;125:86-9. discussion 90.

61. Carver JD. Dietary nucleotides: effects on the immune and gastrointestinal systems. Acta Paediatr Oslo Norway. 1999;1992 Suppl 88:83-8.

62. Eugui EM, Almquist SJ, Muller CD, Allison AC. Lymphocyteselective cytostatic and immunosuppressive effects of mycophenolic acid in vitro: role of deoxyguanosine nucleotide depletion. Scand J Immunol. 1991;33:161-73.

63. Tian H, Cronstein BN. Understanding the mechanisms of action of methotrexate: implications for the treatment of rheumatoid arthritis. Bull NYU Hosp Jt Dis. 2007;65:168-73.

64. Chan ESL, Cronstein BN. Mechanisms of action of methotrexate. Bull Hosp Jt Dis. 2013;2013(71 Suppl 1):S5-8.

65. Courtemanche C, Elson-Schwab I, Mashiyama ST, et al. Folate deficiency inhibits the proliferation of primary human CD8+ T lymphocytes in vitro. J Immunol Baltim Md 1950. 2004;173: 3186-92.

66. Ko IK, Kim B-G, Awadallah A, et al. Targeting improves MSC treatment of inflammatory bowel disease. Mol Ther J Am Soc Gene Ther. 2010;18:1365-72. doi:10.1038/mt.2010.54.

67. MacFarlane AJ, Behan NA, Matias FMG, et al. Dietary folate does not significantly affect the intestinal microbiome, inflammation or tumorigenesis in azoxymethane-dextran sodium sulphate-treated mice. Br J Nutr. 2013;109:630-8. doi:10.1017/S0007114512001857.

68. Rostom A, Dube C, Lewin G. Use of aspirin and NSAIDs to prevent colorectal cancer. Rockville: Agency for Healthcare Research and Quality (US); 2007.

69. Baggott JE, Morgan SL, Ha T, et al. Inhibition of folate-dependent enzymes by non-steroidal anti-inflammatory drugs. Biochem J. 1992;282(Pt 1):197-202.

70. Docherty JG, McGregor JR, O’Dwyer PJ, Galloway DJ. Local recurrence of colorectal cancer: the problem, mechanisms management and adjuvant therapy. Br J Surg. 1994;81:1082.

71. Choumenkovitch SF, Selhub J, Wilson PWF, et al. Folic acid intake from fortification in United States exceeds predictions. J Nutr. 2002;132:2792-8.

72. Bailey RL, Dodd KW, Gahche JJ, et al. Total folate and folic acid intake from foods and dietary supplements in the United States: 2003-2006. Am J Clin Nutr. 2010;91:231-7. doi:10.3945/ajcn. 2009.28427.

73. Shane B (1989) Folylpolyglutamate synthesis and role in the regulation of one-carbon metabolism. In: Aurbach GD, McCormick DB, editors. Vitam Horm. Academic Press, pp 263-335.

74. Ahuja N, Li Q, Mohan AL, et al. Aging and DNA methylation in colorectal mucosa and cancer. Cancer Res. 1998;58:5489-94.

75. Varela-Rey M, Woodhoo A, Martinez-Chantar M-L, et al Alcohol, DNA methylation, and cancer. Alcohol Res Curr Rev. 2013;35:25-35. 